\title{
CHARACTERISTICS OF A NEW RESPIRATORY SYNDROME ASSOCIATED WITH THE USE OF A HUMIDIFIER DISINFECTANT: HUMIDIFIER DISINFECTANT-RELATED RESPIRATORY SYNDROME (HDRS)
}

JONG HAN LEEM ${ }^{1}$, JOON-SUNG JOH ${ }^{2}$, YOUNG-SEOUB HONG ${ }^{3}$, JUNGWON KIM ${ }^{4}$, SOYOUNG PARK ${ }^{5}$, SOYEOUN LIM ${ }^{6}$, and YANGHO KIM ${ }^{7}$

${ }^{1}$ Inha University College of Medicine, Incheon, South Korea

Department of Occupational and Environmental Medicine

${ }^{2}$ National Medical Center, Seoul, South Korea

Department of Internal Medicine

${ }^{3}$ College of Medicine, Dong-A University, Busan, South Korea

Department of Preventive Medicine

${ }^{4}$ Kosin University College of Medicine, Busan, South Korea

Department of Occupational and Environmental Medicine

${ }^{5}$ Sungkyungkwan University, Seoul, South Korea

Kangbuk Samsung Hospital, School of Medicine, Total Health Care Center

${ }^{6}$ University of Ulsan College of Medicine, Ulsan, South Korea

Ulsan University Hospital, Department of Radiology

${ }^{7}$ University of Ulsan College of Medicine, Ulsan, South Korea

Ulsan University Hospital, Department of Occupational and Environmental Medicine

\begin{abstract}
Objectives: The purpose of this study was to characterize a new respiratory syndrome associated with exposure to a humidifier disinfectant (HD) in South Korea that is distinct from the well-known HD-related lung injury (HDLI). The authors identified this condition in 24 study subjects who were family members of patients with definite or probable HDLI (referred to as index cases), and were exposed to HD in the same room as the index cases. Material and Methods: The authors reviewed medical records of 236 family members in 110 families who were exposed to HD in the same rooms and residences as the index cases. Results: They identified 24 family members who were exposed to HD in the same rooms and residences as the index cases, and who developed respiratory disorders that were distinct from HDLI. The clinical signs and symptoms of these individuals were in the upper respiratory tract, such as allergic rhinitis and croup, or in the lower respiratory tract, such as bronchitis and pneumonia. The diffusing capacity of the lung for
\end{abstract}

This work was supported by the National Institute of Environment Research (NIER) of the Republic of Korea (NIER-2018-04-02-075).

Received: January 9, 2020. Accepted: July 28, 2020.

Corresponding author: Yangho Kim, University of Ulsan College of Medicine, Ulsan University Hospital, Department of Occupational and Environmental Medicine, 290-3 Cheonha-dong, Dong-gu, 44033 Ulsan, South Korea (e-mail: yanghokm@ulsan.ac.kr). 
carbon monoxide was reduced in 9 of 12 children (data not available for 1 child), and in 4 of 5 adults (data not available for 6 adults). The percent forced vital capacity and forced expiratory volume in one second/forced vital capacity ratios were within the normal ranges in most patients. The computed tomography findings, which mostly indicated non-specific inflammation, were inconsistent with the radiological diagnostic criteria for HDLI, but were abnormal in 8 of 11 adults, and in 2 of 13 children. Conclusions: The authors propose a new condition, i.e., HD-related respiratory syndrome (HDRS), which is characterized by mild to moderate or atypical respiratory symptoms and signs, and is related to HD exposure, but is distinct from HDLI. The recognition of HDRS may provide a basis for understanding the natural history of HD-related respiratory problems, and for capturing the whole spectrum of HD-related clinical manifestations in the respiratory tract. Int J Occup Med Environ Health. 2020;33(6):829-39

Key words:

pneumonitis, respiratory, lung, disinfection, injuries, humidifier

\section{INTRODUCTION}

Humidifier disinfectant-related lung injury (HDLI) is a serious $\mathrm{HD}$-induced damage to the lungs that occurred exclusively in South Korea and was first reported in 2011 [1]. An antimicrobial biocide, polyhexamethylene guanidine (PHMG) phosphate, was the causative ingredient in the commercially available product [1], and it was subsequently banned. The symptoms of HDLI were cough, dyspnea, and occasionally fever, and the progression was subacute or rapid, similar to acute respiratory distress syndrome (ARDS). Radiologically, HDLI is characterized by diffuse centrilobular ground-glass opacity and nodules, which spares the subpleural spaces and often accompanies spontaneous air leaks, such as pneumothorax or pneumomediastinum, but with no evidence of air trapping or reticular opacity. The histopathological features typically include a bronchocentric distribution of fibroinflammatory lesions, which become more pronounced over time. Epidemiological studies have indicated that HDLI occurs during dry seasons and is clustered within families [2-6]. A rodent study has reported that intratracheal instillation or inhalation of PHMG phosphate induces severe lung inflammation and fibrosis, similar to the pathological features in affected humans [7-9]. The aerosol form of PHMG (nano-sized PHMG) that is released by humidifiers can penetrate and accumulate deep in the lungs [10]. The analysis of the pathological, radiological and clinical features of HDLI cases, and lung histopathology of HD-exposed experimental animals, led to the refinement of the diagnostic criteria for HDLI in 2013 [11,12] (Tables 1 and 2). In the determi- nation of the HD-relatedness of lung diseases, these criteria focused on rapidly progressive damage and severe interstitial lung disease with interstitial fibrosis on the terminal bronchioles and alveoli that can be differentiated from other interstitial lung diseases, such as acute interstitial pneumonia and hypersensitivity pneumonitis (HP). However, these criteria are likely too restrictive to cover the whole spectrum of HD-related respiratory symptoms, which likely include mild or atypical forms of interstitial lung disease and lung parenchymal diseases other than interstitial lung diseases, or upper airway injuries [12].

The authors examined the clinical features of family members of patients with definite or probable HDLI (referred to as index cases) to clarify the whole spectrum of respiratory symptoms and signs related to exposure to HD. All family members were exposed to HD in the same rooms and residences as the HDLI patients.

\section{MATERIAL AND METHODS}

The authors used data from the Korea Environmental Industry and Technology Institute (KEITI), which officially collected information on individuals with lung disease who claimed that their disease was related to the use of HD. The Korean government sent a notice about the nationwide investigation to all persons who had reported or had simply raised concerns about an illness or death potentially due to the use of $\mathrm{HD}$, via an internet webpage, mass media or advertisements providing contact telephone numbers. All registered claimants were asked to submit all relevant medical records, including radiographic images and pa- 
Table 1. Diagnostic criteria for humidifier disinfectant-related lung injury (HDLI) [15]

\begin{tabular}{|c|c|}
\hline Criteria & Lung injury \\
\hline Clinical & $\begin{array}{l}\text { - strong clinical history of symptoms, physical signs, and radiological features consistent with humidifier disinfect- } \\
\text { ant exposure without evidence of infectious, autoimmune and other typical interstitial lung diseases }\end{array}$ \\
\hline CT imaging & $\begin{array}{l}\text { - initially multifocal, patchy consolidation sparing the subpleural areas, then with the disappearance of consolida- } \\
\text { tion followed by progression to diffuse, centrilobular, ground-glass opacity } \\
\text { - no evidence of air trapping or reticular opacity } \\
\text { - persistent, diffuse and extensive centrilobular ground-glass nodular opacities with no evidence of air trapping } \\
\text { on follow-up HRCT }\end{array}$ \\
\hline Pathological & $\begin{array}{l}\text { - non-suppurative necrotizing and obliterate bronchiolitis with various stages of peribronchiolar organizing } \\
\text { alveolar damage } \\
\text { - relative sparing of the subpleural and paraseptal parenchyma } \\
\text { - patchy distribution of alveolar damage with predominant centrilobular distribution } \\
\text { - cellular and fibrosing interstitial pneumonia with predominant centrilobular distribution but without granuloma }\end{array}$ \\
\hline
\end{tabular}

CT - computed tomography; HRCT - high-resolution computed tomography.

Copyright $\odot$ (2017) Korean Society of Environmental Health and Toxicology (KOSEHT). From Environmental Health and Toxicology; by Leem JH, Lee JH. Reprinted by permission of KOSEHT.

Table 2. Classification of injuries in patients with different levels of humidifier disinfectant-related lung injury (HDLI) [12]

\begin{tabular}{cl}
\hline Level & \multicolumn{1}{c}{ Injury classification } \\
\hline 1 (definite) & $\begin{array}{l}\text { as a confirmed case of humidifier disinfectant exposure, the case can be verified through centrilobular radiological } \\
\text { findings, typical clinical findings on the basis of the clinical course of the disease, and/or pathological findings; } \\
\text { the possibility of HDLI (disease of the terminal bronchiole caused by a humidifier disinfectant) is almost certain } \\
\text { or highly probable without any other causes for lung disease } \\
\text { as a confirmed case of humidifier disinfectant exposure, the case can be verified through centrilobular radiological } \\
\text { findings, typical clinical findings on the basis of the clinical course of the disease, and/or pathological findings; } \\
\text { the possibility of HDLI (disease of the terminal bronchiole caused by humidifier disinfectant) is probable } \\
\text { or somewhat likely even though other causes cannot be ruled out completely }\end{array}$ \\
& $\begin{array}{l}\text { as a confirmed case of humidifier disinfectant exposure, the case can be suspected through centrilobular } \\
\text { radiological findings, typical clinical findings on the basis of the clinical course of the disease, and/or pathological } \\
\text { findings, but those findings are not compatible with typical HDLI; the possibility of HDLI (disease of the terminal } \\
\text { bronchiole caused by humidifier disinfectant) is not probable because other causes cannot be ruled out } \\
\text { as a confirmed case of humidifier disinfectant exposure, the case is different from centrilobular radiological } \\
\text { findings, typical clinical findings on the basis of the clinical course of the disease, and/or pathological findings; } \\
\text { the possibility of HDLI (disease of the terminal bronchiole caused by humidifier disinfectant exposure) is almost } \\
\text { certainly unlikely because other causes may be suspected }\end{array}$ \\
lack of data to identify HDLI
\end{tabular}

Copyright $@$ (2016) Korean Society of Environmental Health and Toxicology (KOSEHT). From Environmental Health and Toxicology; by Choi JE, Hong SB, Do KH, Kim HJ, Chung S, Lee E, et al. Reprinted by permission of KOSEHT.

thology specimens to KEITI. The claimants were then clinically examined by the Lung Injury Investigation Committee in KEITI for the diagnosis and confirmation of HDLI. The committee periodically held multidisciplinary con- ferences that were attended by adult and pediatric pulmonologists, radiologists, pathologists, and environmental medicine specialists. They closely reviewed the clinical manifestations, high-resolution computed tomography 
a)

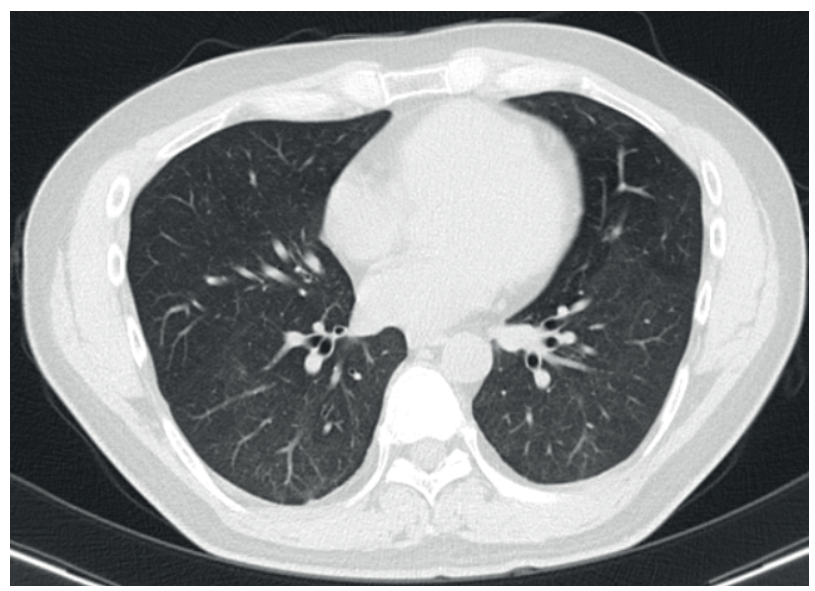

b)

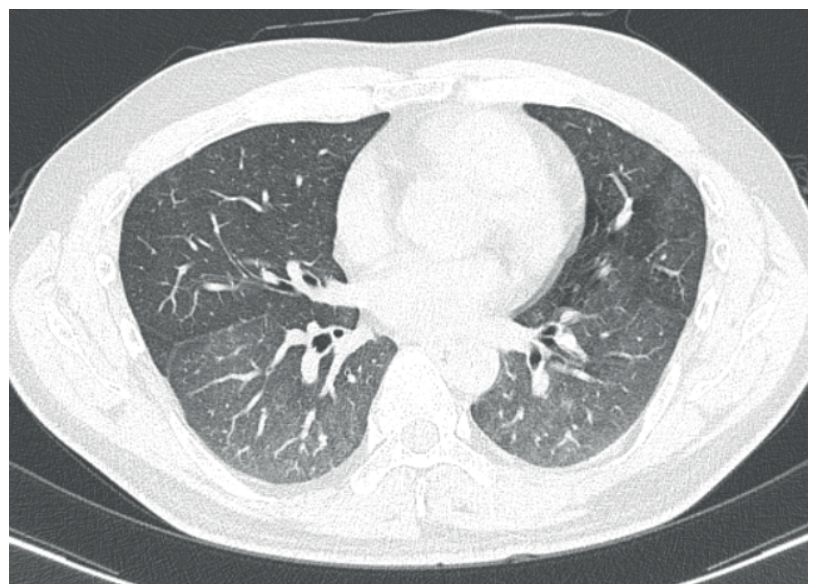

Figure 1. High-resolution computed tomography in a patient of the Asan Medical Center performed on July 15, 2016 a) inspiratory (inhomogeneous lung opacity is visible), and b) expiratory (marked accentuation of inhomogeneity due to air trapping, suggestive of small airway disease) (case 3)

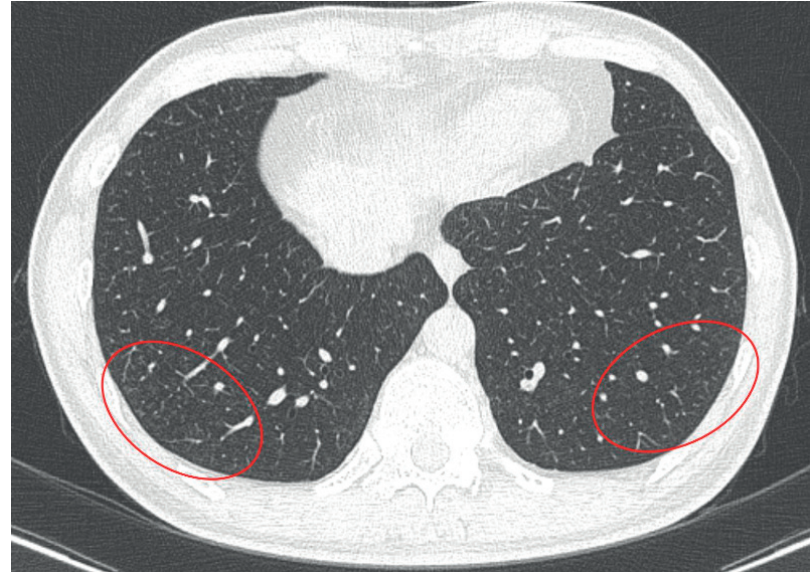

Figure 2. High-resolution computed tomography in a patient of the Asan Medical Center performed on December 8, 2016 regional, subtle tiny centrilobular nodules in bilateral lower lobes (case 4)

(HRCT) observations, and the findings of examinations of various pathology specimens, such as biopsy specimens, and explanted or autopsy lungs of patients in the light of the diagnostic criteria for HDLI (Tables 1 and 2).

The combination of clinical manifestations, radiological findings, exposure to HD, and the temporal relationship between the clinical course and exposure to HD, as well as pathological findings, were used for the confirmation of HDLI. The subjects were selected from the first to third

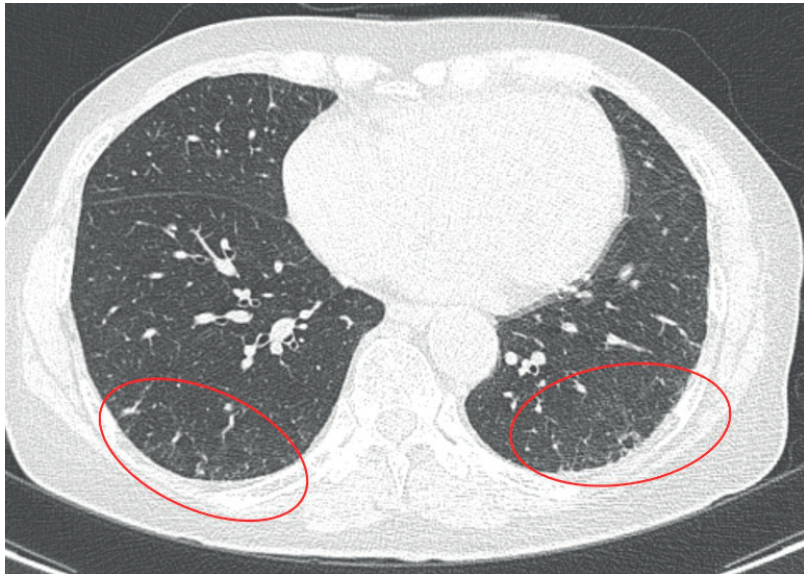

Figure 3. High-resolution computed tomography in a patient of the Asan Medical Center performed on Dec ember 15, 2016 regional, subtle tiny nodules and fine reticulation in bilateral lower lobes (case 5)

round of investigations, conducted in July 2013-December 2015. From the first to third round of investigations, 1196 individuals in 748 families who claimed that their HD exposure was related to their diseases received final decisions from the investigation. At that time, 110 of 748 families had $\geq 1$ members who were recognized as HDLI cases. The authors reviewed the medical records of all the 110 families, defining family member(s) with HDLI as index case(s). They identified 236 family members who were exposed to 
HD in the same rooms and residences as the index cases in these families. After receiving an explanation of the survey, all participants provided written informed consent for participation. This survey was approved by the Institutional Review Board of the National Institute of Environmental Research (NIER-2018-04-02-075).

\section{RESULTS}

The authors identified 24 family members (11 adults and 13 children) with respiratory symptoms, who were exposed to HD in the same rooms and residences as the index cases (Table 3). Their clinical signs and symptoms were compatible with a history of HD exposure, diverse laboratory findings of the diffusing capacity of the lung for carbon monoxide (DLCO), and computed tomography (CT). Their clinical signs and symptoms were in the upper respiratory tract (URT), such as URT infection, allergic rhinitis, croup, or the lower respiratory tract (LRT) infection, such as bronchitis and pneumonia, but were distinct from typical HDLI. Most individuals received care in an outpatient department. The DLCO was reduced in 9 of 12 children (data not available for 1 child), and in 4 of 5 adults (data not available for 6 adults). The percent forced vital capacity (\%FVC) and forced expiratory volume in 1 second/forced vital capacity $\left(\mathrm{FEV}_{1} / \mathrm{FVC}\right)$ ratios were within the normal ranges in almost all patients. The CT findings mostly indicated nonspecific inflammation, and were inconsistent with the radiological diagnostic criteria of HDLI, but were abnormal in 8 of 11 adults and in 2 of 13 children (Figures 1-3).

\section{DISCUSSION}

Generally, HDLI manifests as cough, dyspnea, and occasionally as fever. It usually has a rapidly progressive clinical course, similar to ARDS [2,5], and develops during winter and early spring, the seasons when humidifiers are mainly used in Korea due to low humidity [6]. In numerous cases, HLDI is refractory to steroid therapy. Radiologically, HLDI is characterized by diffuse centrilobular ground-glass opacity and nodules sparing of subpleural spaces, often with spontaneous air leaks, such as pneumothorax or pneumomediastinum, but with no evidence of air trapping or reticular opacity.

The differential diagnosis should differentiate HDLI from HP and acute exacerbation of idiopathic pulmonary fibrosis (IPF). Subacute HP shows characteristic radiological findings, such as mosaic attenuation due to air trapping; this is rare in HDLI, although HP also shows ill-defined centrilobular opacities [13]. Moreover, the lack of response to corticosteroid therapy is inconsistent with a diagnosis of HP [2]. Pathologically, HP is characterized by bronchiolocentric granulomatous lymphocytic alveolitis, whereas HDLI is characterized by diffuse alveolar damage [11]. Acute exacerbation of IPF often presents as a rapid progression of respiratory failure, similar to HDLI, but a bronchocentric distribution of lung injury is inconsistent with IPF [4]. A bronchocentric injury pattern suggests inhalation of HD as the causative mechanism [4].

In the present study, approximately $10 \%$ of the study subjects presented with a variety of respiratory symptoms and signs, such as rhinitis, sinusitis, pharyngitis, laryngitis, asthma, bronchitis, bronchiolitis, and pneumonia, that were inconsistent with HDLI, defined as severe interstitial lung disease focused on the terminal bronchioles and alveoli. However, each of the 24 subjects had family members with confirmed HDLI, so it seemed likely that these subjects also had a high level of exposure to HD. The spectrum of HD-related respiratory injuries included lung parenchymal diseases other than interstitial lung diseases, and URT injuries. Thus, the respiratory effects of HD exposure include respiratory diseases such as rhinitis, sinusitis, pharyngitis, laryngitis, asthma, bronchitis, bronchiolitis and pneumonia, as well as HDLI, the most severe and (sub)acute form of lung damage.

The present study suggests that other atypical signs, in addition to centrilobular radiological findings used as a criterion of HDLI, can occur in HD-related lung injuries. 


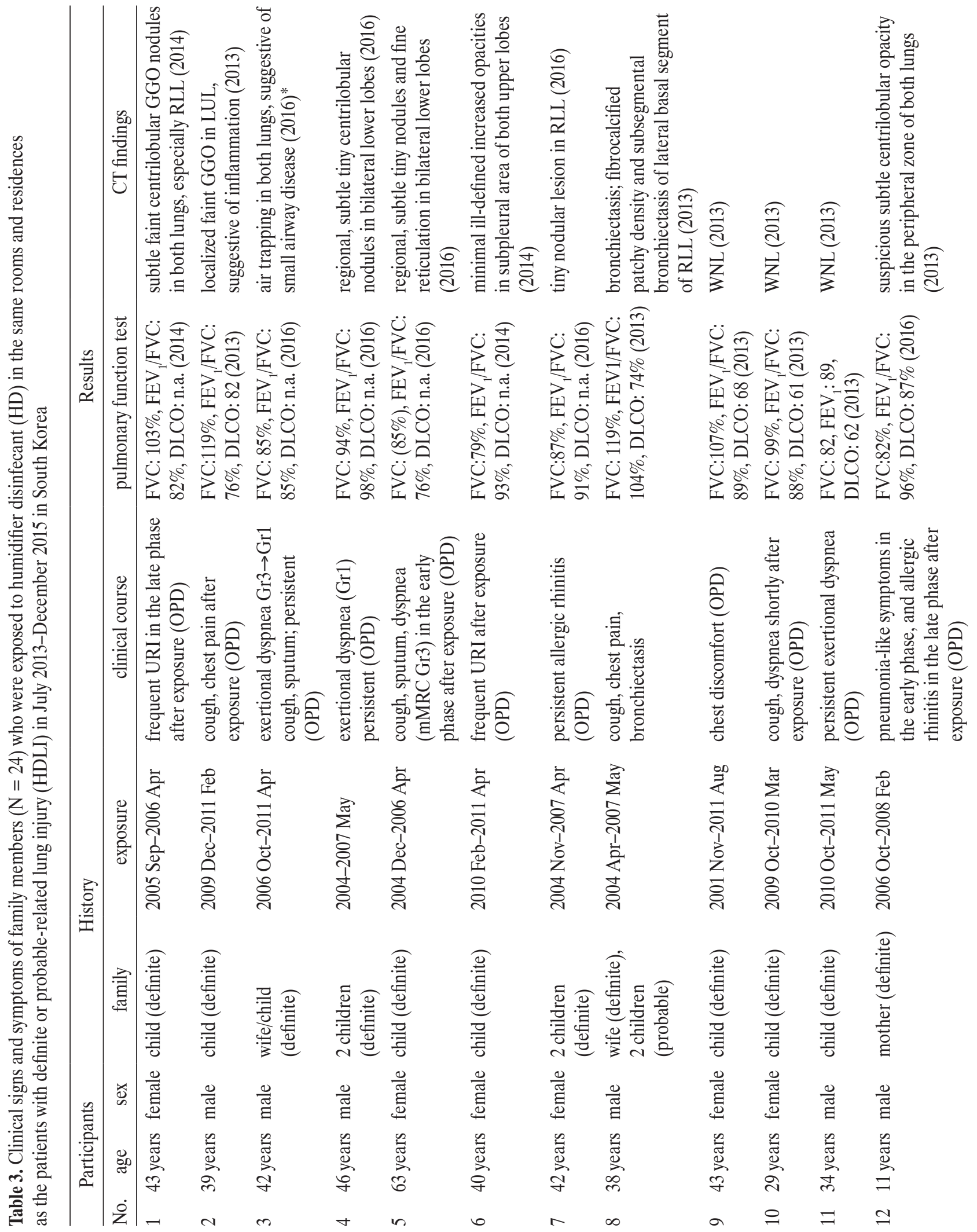




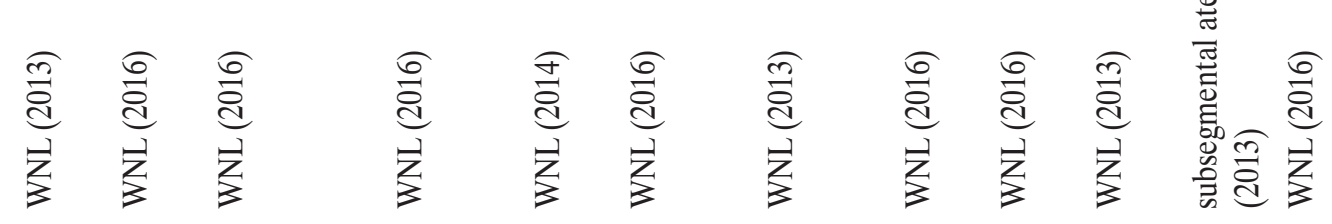

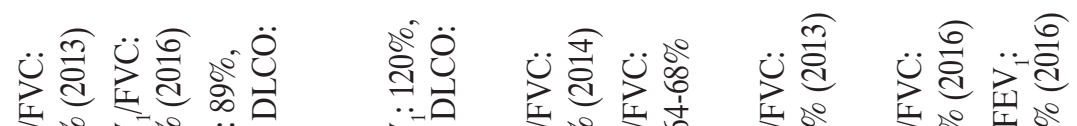

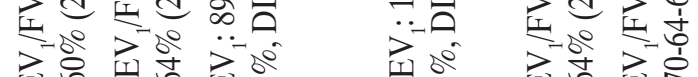

$>0$

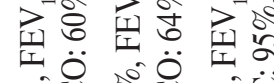

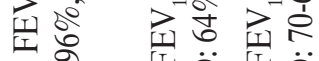

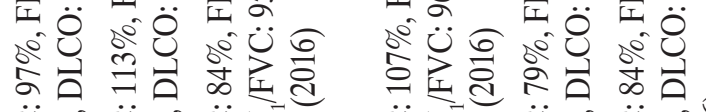

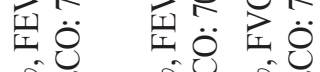

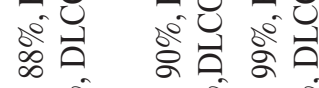

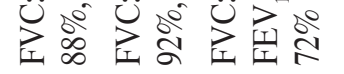

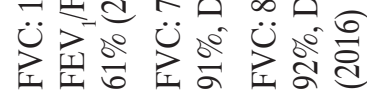

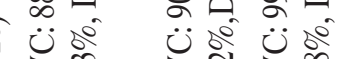

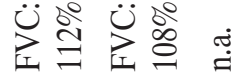

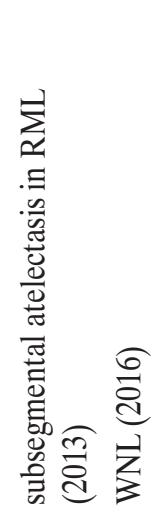

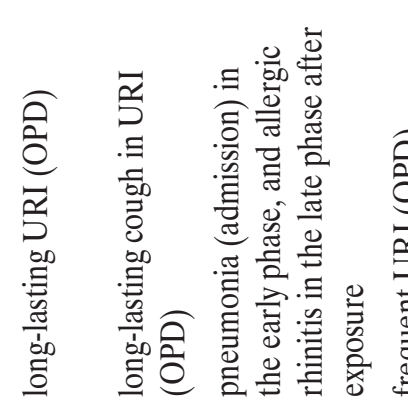
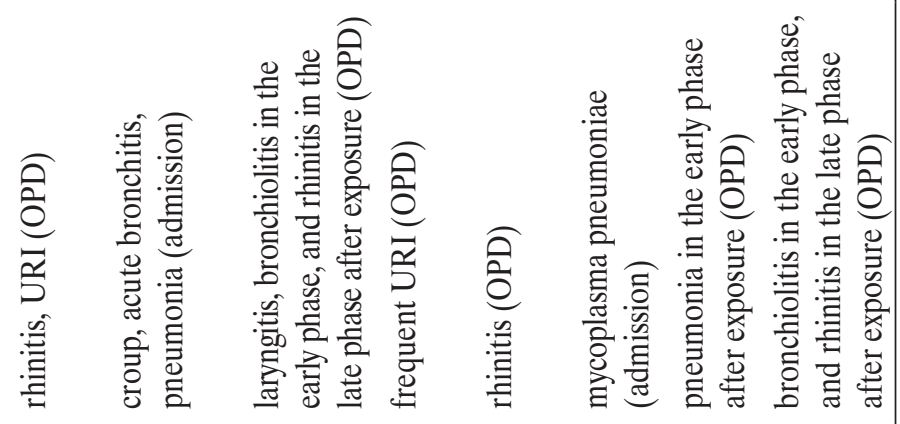

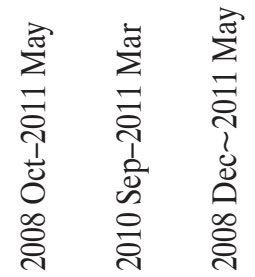

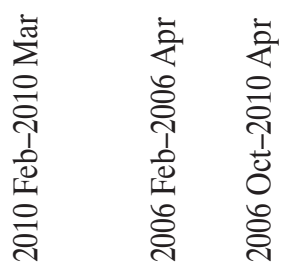

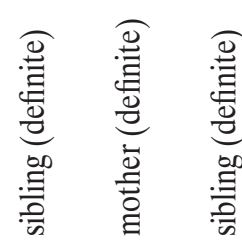

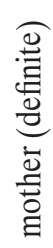

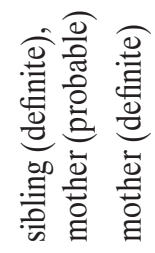

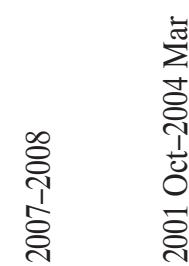

敢

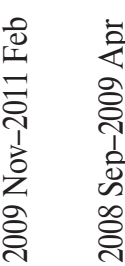

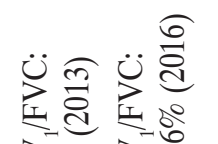

$\begin{array}{ll}> & >0 \\ 1 & 0\end{array}$

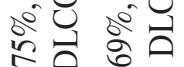

نें

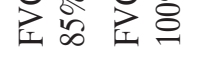

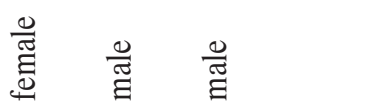

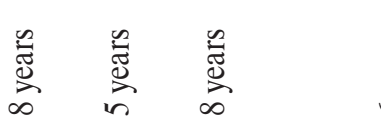

m

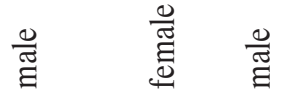

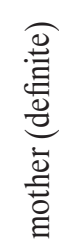

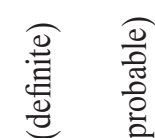

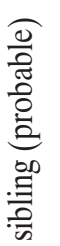

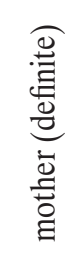

今ั

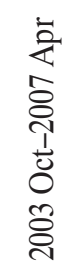

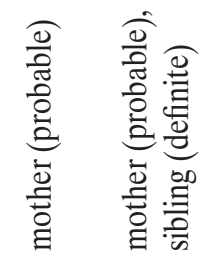

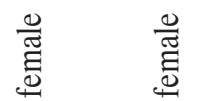

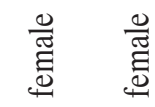

苞 莺

芯 离

芯

$\stackrel{心}{\stackrel{\varpi}{*}}$

离 䞤

(⿻)

2

ำ

$\vec{\pi}$ 
The HRCT findings of the individuals exposed to HD vary with the disease severity and the time since exposure. Those who did not survive HDLI more often had spontaneous air leaks, with a wide area of consolidation and diffuse ground-glass opacity, compared with survivors $[4,14]$. The major radiological features of HDLI show unique changes over time. There are initially areas of patchy consolidation involving the upper lung periphery and posterior lower lung zones, but sparing the subpleural areas. These areas evolve into centrilobular opacities with the disappearance of consolidation, and gradually disappear at about 5 years after cessation of exposure [11,15]. A recent paper has shown that HDLI likely has a diverse clinical course, and that emphysema and bronchiectasis can occur in the advanced stages, based on long-term follow-up [1]. As DLCO is a measure of the conductance of gas transfer from inspired gas to the red blood cells [16], when reduced, it may indicate a condition that affects the effective alveolar surface area [17]. A previous study examined 39 HDLI patients who underwent a pulmonary function testing at diagnosis, and reported a restrictive lung disease pattern and reduced DLCO. However, lung function recovered to normal in $54 \%$ of these patients, with the return of mean FVC $(\%)$ values to the normal range, whereas the mean DLCO remained low during follow-up [1]. Thus, reduced DLCO may be considered a marker of lung injury. Furthermore, many of the present 24 patients who experienced previous respiratory symptoms and signs had low DLCO, even though their \%FVC and CT results were normal. Hence, DLCO may be a useful biomarker of a past lung injury from HDLI in individuals whose current pulmonary function test and CT results are normal.

The clinical spectrum of HD-related respiratory symptoms and signs appears to range from asymptomatic to full-blown acute respiratory failure and death [3,11]. Thus, some family members who were exposed to HD in the same rooms as HDLI patients (the index cases) experienced no respiratory symptoms. However, most family members responded to supportive care, such as symptomatic therapy or antibiotic treatment, and they did not fulfill the diagnostic criteria of HDLI. A previous study of HDLI reported that patients who experienced mild hypoxemia responded to supportive care, such as conventional oxygen therapy, antibiotic treatment and steroids [3]. However, patients with refractory hypoxemia exhibited rapid progression despite the administration of antibiotics, antivirals, antifungals, steroids and other immunosuppressants, as well as mechanical ventilation and extracorporeal membrane oxygenation [3]. Most of the index cases in the present study were in this latter category.

Recent research has shown that exposure to HD also increases the risk of asthma and interstitial pneumonitis $[18,19]$. Taken together, it is possible that HD-related respiratory manifestations have clinical features ranging from mild to moderate or atypical upper/lower airway diseases to severe interstitial lung damage, such as HDLI. This interpretation is compatible with the toxicological perspective implying that the severity of a toxicant-related disease depends on the dose, exposure pattern, and host factors. The current diagnostic criteria of HDLI focused on a rapidly progressive and severe lung injury, and were developed to exclude patients whose clinical symptoms may have been similar but were not due to HD exposure. As a result, these criteria excluded many patients who were exposed to HD but only experienced mild to moderate respiratory symptoms or atypical respiratory problems. The present findings suggest that these patients with mild to moderate or atypical respiratory symptoms should be classified as clinical manifestations related to HD exposure.

Thus, the current diagnostic criteria of HDLI consider the causal relationship between severe lung injury and exposure to HD as highly specific, but it is very rare to have highly specific causes of diseases in human toxicology. Instead, most causal relationships in toxicology are nonspecific, in that there may be multiple causes of a single 
disease, and a single factor may cause multiple diseases [20]. In the case of the health effects of HD, a complex combination of causes may lead to a spectrum of effects, from specific to non-specific. This is because the physical characteristics, dose and host factors of a toxicant determine its effects [20].

Therefore, a new classification is needed which is compatible with the natural history and the whole spectrum of toxicant-related diseases. Thus, the authors propose the condition of HD-related respiratory syndrome (HDRS), which is defined by the presence of mild to moderate or atypical respiratory symptoms and signs related to HD exposure, and is distinct from HDLI. The diagnostic criteria for HDRS are:

- a status as a family member of an HDLI index case, who was exposed to HD in the same room and residence;

- mild to moderate or atypical clinical symptoms and signs involving URT and LRT, but distinct from typical HLDI;

- non-specific HRCT findings, distinct from typical centrilobular radiological findings used to diagnose HDLI, or reduced DLCO;

- a history of exposure to HD, clinical manifestations, and CT findings that are compatible.

The present study is the first to evaluate the clinical features of family members of recognized HDLI patients who were also exposed to a high level of HD. Little is known about the natural history or the whole spectrum of HDrelated clinical manifestations in the respiratory tract, except for full-blown HDLI. Therefore, the recognition of HDRS may provide a better understanding of the natural history of HD-related respiratory problems by capturing the whole spectrum of HD-related clinical manifestations in the respiratory tract.

In the present study, the authors identified HDRS as a new type of respiratory syndrome that is distinct from HDLI. The syndrome is characterized by the presence of mild to moderate clinical symptoms and signs involving URT and LRT, and was present in 24 of 236 family members that had an HDLI index case. The differences in clinical manifestations among family members could be explained by differences in exposure to $\mathrm{HD}$, in which exposure to a higher concentration and/or longer duration may lead to HDLI, and by differences in individual susceptibility to PHMG phosphate among family members.

The present study had several strengths. The authors examined family members of recognized HDLI patients (definite or probable) who were exposed to HD in the same room. The cohabitation of a family member with an HDLI patient provides a reliable assessment of a high level of exposure. The present study also had some limitations. First, the authors could not quantify HD exposure, and were, therefore, unable to compare the dose, duration, frequency and cumulative dose, or the effect of shortterm high-level exposure to HD, among family members although they limited the study subjects to those family members who were exposed to HD in the same rooms as the HDLI (a homogenous exposure group). Second, they did not examine differences in susceptibility between family members. Finally, they had no control population, and were unable to compare these results with those in the control population.

\section{CONCLUSIONS}

The authors identified 24 family members of HDLI index cases who were exposed to HD in the same rooms and residences as the index cases. These family members showed respiratory signs or symptoms that were distinct from typical HDLI. Thus, the authors propose a new disease, HDRS, defined by the presence of mild to moderate or atypical respiratory symptoms and signs related to HD exposure, that is distinct from HDLI. The recognition of HDRS may improve the overall understanding of the natural history of HD-related respiratory problems by capturing the whole spectrum of HD-related clinical manifestations in the respiratory tract. 


\section{REFERENCES}

1. Huh JW, Hong SB, Do KH, Koo HJ, Jang SJ, Lee MS, et al. Inhalation Lung Injury Associated with Humidifier Disinfectants in Adults. J Korean Med Sci. 2016;31(12):1857-62, https://doi.org/10.3346/jkms.2016.31.12.1857.

2. Lee E, Seo JH, Kim HY, Yu J, Jhang WK, Park SJ, et al. Toxic inhalational injury-associated interstitial lung disease in children. J Korean Med Sci. 2013;28(6):915-23, https:// doi.org/10.3346/jkms.2013.28.6.915.

3. Kim WY, Hong SB. Humidifier Disinfectant-Associated Lung Injury: Six Years after the Tragic Event. Tuberc Respir Dis. 2017;80(4):351-7, https://doi.org/10.4046/trd.2017. 0048.

4. Yoon HM, Lee E, Lee JS, Do KH, Jung AY, Yoon CH, et al. Humidifier disinfectant-associated children's interstitial lung disease: Computed tomographic features, histopathologic correlation and comparison between survivors and non-survivors. Eur Radiol. 2016;26(1):235-43, https://doi. org/10.1007/s00330-015-3813-1.

5. Hong SB, Kim HJ, Huh JW, Do KH, Jang SJ, Song JS, et al. A cluster of lung injury associated with home humidifier use: clinical, radiological and pathological description of a new syndrome. Thorax. 2014;69(8):694-702, https://doi. org/10.1136/thoraxjnl-2013-204135.

6. Park JH, Kim HJ, Kwon GY, Gwack J, Park YJ, Youn SK, et al. Humidifier Disinfectants Are a Cause of Lung Injury among Adults in South Korea: A Community-Based CaseControl Study. PloS One. 2016;11(3):e0151849, https://doi. org/10.1371/journal.pone.0151849.

7. Kim HR, Lee K, Park CW, Song JA, Shin DY, Park YJ, et al. Polyhexamethylene guanidine phosphate aerosol particles induce pulmonary inflammatory and fibrotic responses. Arch Toxicol. 2016;90(3):617-32, https://doi.org/10.1007/ s00204-015-1486-9.

8. Song J, Kim W, Kim YB, Kim B, Lee K. Time course of polyhexamethyleneguanidine phosphate-induced lung inflammation and fibrosis in mice. Toxicol Appl Pharmacol. 2018;345:94-102, https://doi.org/10.1016/j.taap.2018.02.013.
9. Song JA, Park HJ, Yang MJ, Jung KJ, Yang HS, Song CW, et al. Polyhexamethyleneguanidine phosphate induces severe lung inflammation, fibrosis, and thymic atrophy. Food Chem Toxicol. 2014;69:267-75, https://doi.org/10.1016/j.fct.2014.04.027.

10. Lee JH, Yu IJ. Human exposure to polyhexamethylene guanidine phosphate from humidifiers in residential settings: Cause of serious lung disease. Toxicol Ind Health. 2017;33(11):83542, https://doi.org/10.1177/0748233717724983.

11. Paek D, Koh Y, Park DU, Cheong HK, Do KH, Lim CM, et al. Nationwide Study of Humidifier Disinfectant Lung Injury in South Korea, 1994-2011. Incidence and Dose-Response Relationships. Ann Am Thorac Soc. 2015;12(12):1813-21, https://doi.org/10.1513/AnnalsATS.201504-2210C.

12. Choi JE, Hong SB, Do KH, Kim HJ, Chung S, Lee E, et al. Humidifier disinfectant lung injury, how do we approach the issues? Environ Health Toxicol. 2016;31:e2016019, https://doi.org/10.5620/eht.e2016019.

13. Selman M, Pardo A, King TE, Jr. Hypersensitivity pneumonitis: insights in diagnosis and pathobiology. Am J Respir Crit Care Med. 2012;186(4):314-24, https://doi.org/10.1164/ rccm.201203-0513CI.

14. Koo HJ, Do KH, Chae EJ, Kim HJ, Song JS, Jang SJ, et al. Humidifier disinfectant-associated lung injury in adults: Prognostic factors in predicting short-term outcome. Eur Radiol. 2017;27(1):203-11, https://doi.org/10.1007/s00330-0164367-6.

15. Leem JH, Lee JH. Humidifier disinfectant-associated specific diseases should be called together as "humidifier disinfectant syndrome”. Environ Health Toxicol. 2017;32:e2017017, https://doi.org/10.5620/eht.e2017017.

16. Sue DY, Oren A, Hansen JE, Wasserman K. Diffusing capacity for carbon monoxide as a predictor of gas exchange during exercise. N Engl J Med. 1987;316(21):1301-6, https:// doi.org/10.1056/nejm198705213162103.

17. Johnson DC. Importance of adjusting carbon monoxide diffusing capacity (DLCO) and carbon monoxide transfer coefficient (KCO) for alveolar volume. Respir Med. 2000;94(1):28-37, https://doi.org/10.1053/rmed.1999.0740. 
18. Yoon J, Lee SY, Lee SH, Kim EM, Jung S, Cho HJ, et al. Exposure to Humidifier Disinfectants Increases the Risk of Asthma in Children. Am J Respir Crit Care Med. 2018;198(12):15836, https://doi.org/10.1164/rccm.201805-0840LE.

19. Lamichhane DK, Leem JH, Lee SM, Yang HJ, Kim J, Lee JH, et al. Family-based case-control study of exposure to household humidifier disinfectants and risk of idiopathic interstitial pneumonia. PloS One. 2019;14(9):e0221322, https://doi.org/10.1371/journal.pone.0221322.

20. Kim S, Paek D. Humidifier disinfectant disaster: what is known and what needs to be clarified. Environ Health Toxicol. 2016;31:e2016025, https://doi.org/10.5620/eht.e2016025.

This work is available in Open Access model and licensed under a Creative Commons Attribution-NonCommercial 3.0 Poland License - http://creativecommons.org/ licenses/by-nc/3.0/pl/deed.en. 